\title{
Resolution of the Phytanic Acid $\alpha$-Oxidation Pathway: Identification of Pristanal as Product of the Decarboxylation of 2-Hydroxyphytanoyl-CoA
}

\author{
N. M. Verhoeven,* D. S. M. Schor,* H. J . ten Brink,* R. J . A. Wanders,† and C. J akobs*,1 \\ *Department of Clinical Chemistry, Free University Hospital, Amsterdam, The Netherlands; and †Departments of Clinical \\ Chemistry and Pediatrics, University of Amsterdam, Academic Medical Centre, Amsterdam, The Netherlands
}

Received J une 26, 1997

The structure and enzymology of the phytanic acid $\alpha$-oxidation pathway have long remained an enigma. Recent studies have shown that phytanic acid first undergoes activation to its coenzyme $A$ ester, followed by hydroxylation to 2-hydroxyphytanoyl-CoA. In this paper we have studied the mechanism of decarboxylation of 2-hydroxyphytanoyl-CoA in human liver. To this end, human liver homogenates were incubated with 2-hydroxyphytanoyl-CoA in the presence or absence of $\mathrm{NAD}^{+}$. Hereafter, the medium was analyzed for the presence of pristanal and pristanic acid by gas chromatography mass spectrometry. Our results show that pristanal is formed from 2-hydroxyphytanoylCoA. Pristanal is subsequently oxidized to pristanic acid in a NAD ${ }^{+}$dependent reaction. These results finally resolve the mechanism of the phytanic acid $\alpha$ oxidation process in human liver. 1997 Academic Press

Phytanic acid $(3,7,11,15$-tetramethylhexadecanoic acid) is first metabolised by $\alpha$-oxidation, as $\beta$-oxidation is blocked by the methyl group in the 3-position. The product of $\alpha$-oxidation is pristanic acid (2,6,10,14-tetramethylpentadecanoic acid), which can be further metabolised by peroxisomal $\beta$-oxidation. The elucidation of the mechanism and the subcellular localisation of the $\alpha$-oxidation process have taken many years of debate in literatureal ready. However, recent data have shed new light on the pathway of phytanic acid $\alpha$-oxidation. It is now known that, after activation to phytanoyl-CoA, 2hydroxyphytanoyl-CoA is formed in a reaction catalysed by phytanoyl-CoA hydroxylase[1,2,3]. This dioxygenase type of enzyme requires $\mathrm{Fe}^{2+}$ and ascorbate as cofactors and $\alpha$-ketoglutarate as cosubstrate, as was

\footnotetext{
${ }^{1}$ Corresponding author. Metabolic Unit, Free University Hospital, De Boelelaan 1117, 1081 HV, Amsterdam, The Netherlands. Fax: 31 20 4440305. E-mail: secr.metabolicunit@AZVU.NL.
}

shown in both rat and human liver [1,2,3]. PhytanoylCoA hydroxylation was found to be deficient in liver and fibroblasts from patients affected by Refsum disease, rhizomelic chondrodysplasia punctata and generalised peroxisomal disorders like Zellweger syndrome, providing the biochemical basis for the phytanic acid accumulation found in these disorders $[1,4,5,6]$. The mechanism whereby 2-hydroxyphytanoyl-CoA is decarboxylated to pristanic acid or pristanoyl-CoA, has remained unknown so far. A systematic study of the reaction mechanisms of enzymes suggested to us the possibility that the decarboxylation of 2-hydroxyphytanoyl-CoA might occur via a mechanism resembling the breakdown of 3-hydroxy-3-methylglutaryl-CoA (HMGCOA). This metabolite is converted into acetoacetate and acetyl-CoA by means of HMG-CoA lyase [7]. If a similar enzyme would be operative in the breakdown of 2-hydroxyphytanoyl-CoA, one would expect formation of pristanal as intermediary metabolite next to formyl-CoA. Further $\mathrm{NAD}^{+}$dependent oxidation of pristanal to pristanic acid could occur by a similar mechanism as described for other fatty aldehydes [8].

In this paper we show that pristanal is produced from 2-hydroxyphytanoyl-COA and undergoes further $\mathrm{NAD}^{+}$dependent oxidation to pristanic acid. Our data lead us to conclude that thelong-sought structure of the phytanic acid $\alpha$-oxidation pathway is finally resolved, after more than 25 years of study.

\section{MATERIALS AND METHODS}

Materials. 2-Hydroxyphytanic acid, pristanic acid and [2-methyl${ }^{2} \mathrm{H}_{3}$ ]pristanic acid were synthesized as described before [9]. 2- $\mathrm{Hy}-$ droxyphytanoyl-CoA was synthesised from 2-hydroxyphytanic acid using the mixed anhydride method [10].

Pristanal ethoxime was synthesized in the F ree University Hospital Amsterdam with a purity of $>99 \%$. Dodecylaldehyde (purity $>96 \%$ ) was obtained from J anssen Chimica (Belgium).

Methods. Pieces of human liver were stored at $-70^{\circ} \mathrm{C}$ and thawed in a medium containing $250 \mathrm{mM}$ mannitol, $5 \mathrm{mM}$ MOPS-KOH, 0.1 

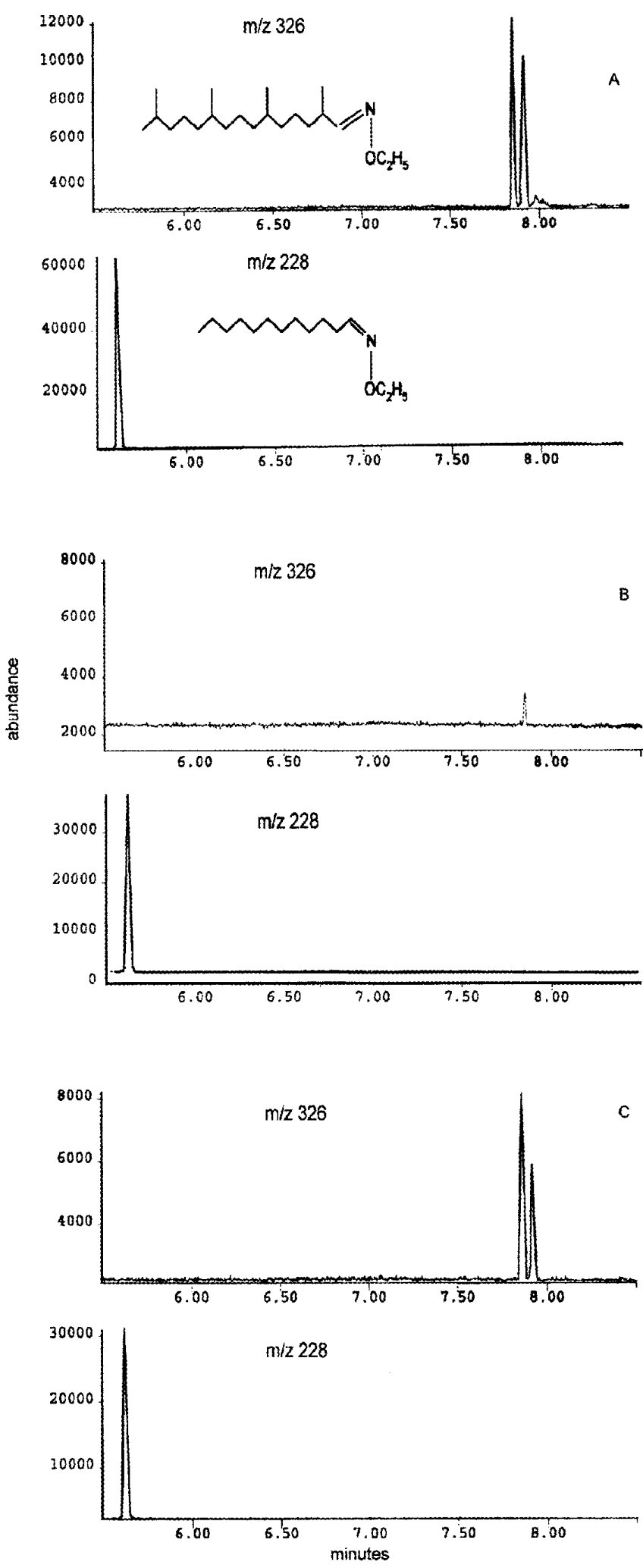

FIG. 1. Mass fragmentogram of (A) a standard solution containing pristanal-ethoxime (authentic compound) and dodecylaldehyde-ethoxime as internal standard, (B) an extract from the incubation medium of human liver homogenate incubated with 2-hydroxyphytanoyl-CoA in the absence of $\mathrm{NAD}^{+}$at $\mathrm{t}=0$ and $(\mathrm{C})$ extract from
$\mathrm{mM}$ EDTA at a final $\mathrm{pH}$ of 7.4. Protein concentrations were determined using an established method [11].

The incubations contained the following components: $1 \mathrm{mg}$ of total liver homogenate, $150 \mathrm{mM} \mathrm{KCl}, 50 \mathrm{mM}$ Hepes- $\mathrm{NaOH}, 2 \mathrm{mM}$ potassium phosphate, $10 \mu \mathrm{M}$ defatted bovine serum al bumin and $0.02 \mathrm{mM}$ 2-hydroxyphytanoyl-CoA. The volume was $1 \mathrm{ml}$ and the final $\mathrm{pH}$ was 7.4. To some of the incubations, $1 \mathrm{mM} \mathrm{NAD}^{+}$was added. $200 \mu \mathrm{l}$ aliquots of the incubations were taken at the start of the reaction and after 10, 30, 60 and 120 minutes. For analysis of pristanal, to these aliquots, $100 \mu$ l ethoxylamine $(0.1 \mathrm{~g} / \mathrm{ml})$ in water was added immediately and the $\mathrm{pH}$ was increased to $>10$ by addition of $\mathrm{Na}_{2} \mathrm{CO}_{3}$. $0.6 \mathrm{nmol}$ dodecylal dehyde was added as internal standard. Hereafter, fatty ethoxime esters were extracted with $2 \mathrm{ml}$ of diethylether by shaking the samples during 1 hour. The ether layers were separated from the aqueous layers and evaporated. The residues were redissolved in $20 \mu$ l of hexane and analyzed by gas chromatography (Hewlett Packard 5890 series II) using a CPsil 19 CB capillary column (24 $\mathrm{m} \times 0.25 \mathrm{~mm}$, film thickness $0.2 \mu \mathrm{M}$, Chrompack, Middelburg, The Netherlands). Detection was performed by positive chemical ionization mass spectrometry (Hewlett Packard Engine 5989B) using methane as reactant gas. For pristanal-ethoxime, $\mathrm{m} / \mathrm{z} 326(\mathrm{M}+1)$ was monitored, for dodecylaldehyde-ethoxime $\mathrm{m} / \mathrm{z} 228(M+1)$ was monitored.

Pristanic acid in the incubations was measured as its pentafluorobenzyl derivative by stable isotope dilution gas chromatography mass spectrometry with negative chemical ionization, essentially as described before for plasma samples [12].

\section{RESULTS}

Figure 1 shows the mass fragmentograms of a standard solution containing pristanal-ethoxime $(\mathrm{Rf}=7.85$ $\min$.) and dodecylaldehyde-ethoxime ( $R f=5.62 \mathrm{~min}$.$) .$ Pristanal-ethoxime gives a double peak in its mass fragmentogram, due to separation between its cis- and trans isomers. No separation between the stereoisomers of dodecylal dehyde-ethoxime is observed.

In incubations of homogenised human liver with 2hydroxyphytanoyl-CoA, formation of pristanal is observed, as is shown in fig. $1 C$. At $t=0$ (fig. 1B), no pristanal was detectable, whereas at $t=100 \mathrm{~min}$. (fig. 1C) a significant amount of pristanal was formed. In these incubations, only a small amount of pristanic acid (0.4 $\mathrm{nmol} / \mathrm{ml}$ ) was formed.

In figure 2 the time dependence of the formation of pristanal in incubations of 2-hydroxyphytanoyl-CoA is shown. A linear increase in pristanal concentrations, as calculated by dividing the peak area of pristanalethoxime by the peak area of the internal standard dodecylaldehyde-ethoxime, was found during the first 60 minutes of incubation. Hereafter, no further increase occurred. In these incubations, to which no $\mathrm{NAD}^{+}$was added, only a small amount of pristanic acid $(0.4 \mathrm{nmol} / \mathrm{ml})$ was formed. When $\mathrm{NAD}^{+}$was added to the incubation medium, there was no detectable formation of pristanal as shown in fig.2. Under these condi-

the same incubation medium at $\mathrm{t}=100$ minutes. F or pristanal-ethoxime $m / z=326(M+1)$ and for dodecylaldehyde-ethoxime $m / z=228$ $(M+1)$ were monitored. 
pristanal (m/z 326) / dodecylaldehyde ( $\mathrm{m} / \mathrm{z} 228)$

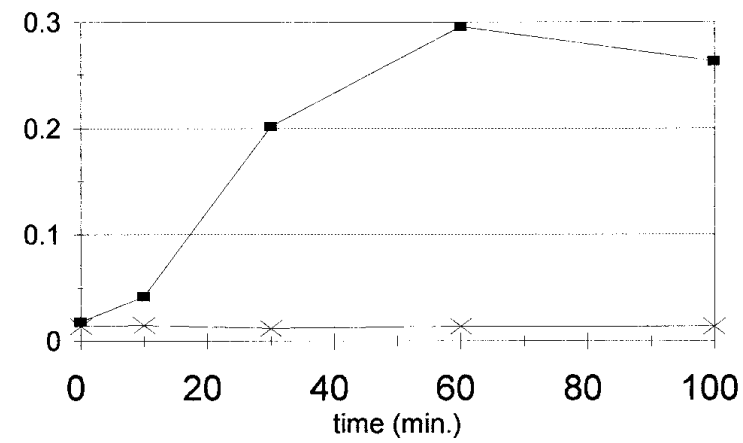

FIG. 2. Formation of pristanal in human liver. Human liver homogenates were incubated with 2-hydroxyphytanoyl-CoA in the presence $(\times)$ or absence $(\boldsymbol{\square})$ of $0.1 \mathrm{mM} \mathrm{NAD}^{+}$. At different time points, $200 \mu \mathrm{l}$ aliquots of the incubation mixture were taken and derivatized with ethoxylamine. The samples were analyzed by gas chromatography mass spectrometry. The amounts of pristanal were quantified relative to the internal standard, dodecylaldehyde.

tions, however, there was a significant formation of pristanic acid $(2.3 \mathrm{nmol} / \mathrm{ml})$.

\section{DISCUSSION}

After long debates in literature about the mechanism of phytanic acid $\alpha$-oxidation in man, the phytanic acid $\alpha$-oxidation pathway has now been resolved. It is evident that the first step involves the activation of phytanic acid to phytanoyl-CoA, catalyzed by a peroxisomal acyl-CoA synthetase al so accepting palmitic acid and pristanic acid as substrates [13]. The second step involves the hydroxylation of phytanoyl-CoA to 2-hydroxyphytanoyl-CoA via a dioxygenase type of enzyme localised in peroxisomes. In this paper we have studied the mechanism by which 2-hydroxyphytanoyl-CoA is further metabolised to pristanic acid. Our data show that the intermediate in this reaction is the fatty aldehyde pristanal (fig. 3). The conversion of 2-hydroxyphytanoyl-CoA into pristanal is probably catalyzed by a lyase type of enzyme, similar to the breakdown of HMG-CoA to acetyl-CoA and acetoacetate by HMGCOA lyase. The recent finding that formyl-COA rather than formic acid is the other product of the decarboxylation process gives support to this idea [14].

Pristanal is converted into pristanic acid in a NAD ${ }^{+}$ dependent reaction, as was illustrated by the absence of pristanal and presence of pristanic acid in incubations to which $\mathrm{NAD}^{+}$was added. Oxidation of fatty aldehydes to their corresponding fatty acids has been described before [8]. Presumably, the formation of pristanal is the limiting step in the overall decarboxylation of 2-hydroxyphytanoyl-CoA, since no pristanal was detectable when further conversion of pristanal was made possible by the addition of $\mathrm{NAD}^{+}$.

There are other examples of $\alpha$-oxidative processes, in which a fatty aldehyde is formed and further oxi- dized to its corresponding fatty acid, in nature. Already in 1959 Martin and Stumpf described the $\alpha$-oxidation of long chain fatty acids in peanuts [15]. A peroxidase enzyme shortens the chain of a fatty acid by one carbon atom, yielding a fatty aldehyde, which is further oxidised by a NAD ${ }^{+}$specific dehydrogenase giving the corresponding fatty acid. This process, however, was then found to be independent of activation of the fatty acids to their coenzyme A derivatives.

The data described in this paper suggest that the pathway of phytanic acid $\alpha$-oxidation is finally resolved now. Following earlier findings that phytanic acid first undergoes activation to phytanoyl-CoA succeeded by hydroxylation to 2-hydroxyphytanoyl-CoA, our data now
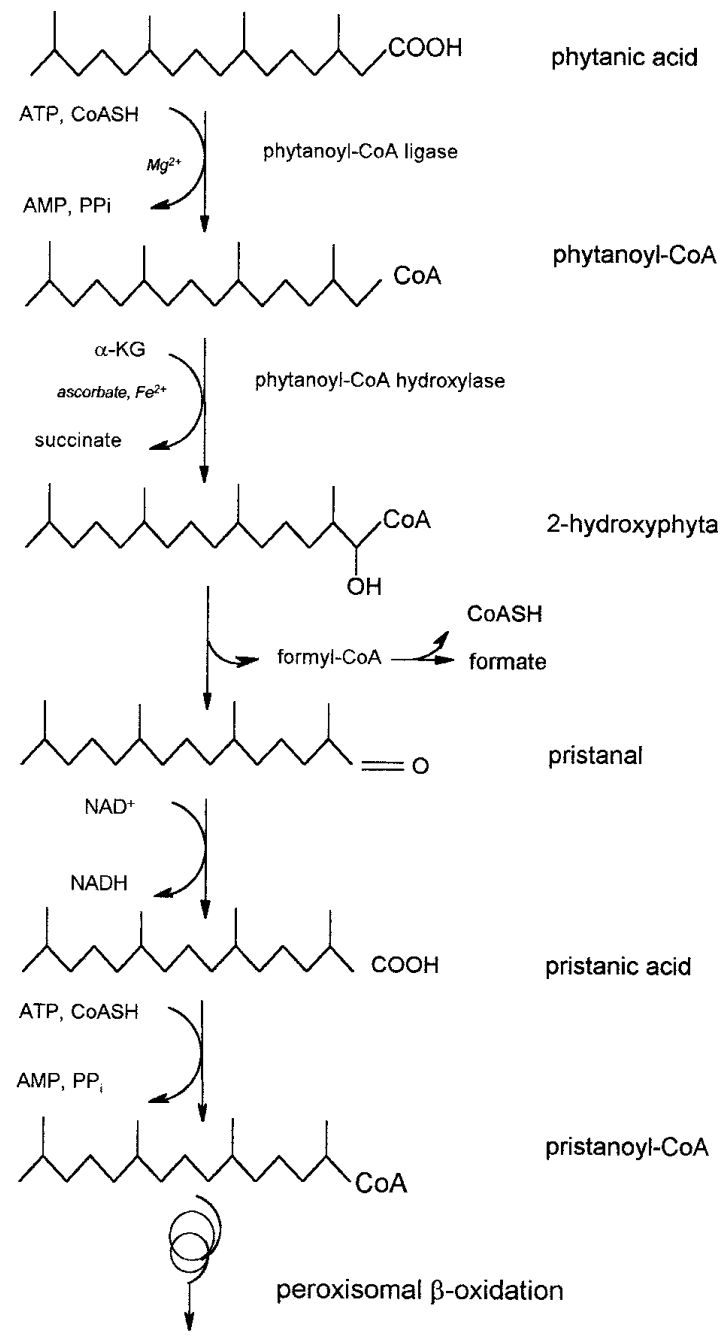

FIG. 3. Schematic representation of the phytanic acid $\alpha$-oxidation pathway in human liver. After activation to phytanoyl-CoA, 2hydroxyphytanoyl-CoA is formed by phytanoyl-CoA hydroxylase, a dioxygenase type of enzyme. Hereafter, 2-hydroxyphytanoyl-CoA is converted into pristanal and formyl-CoA is released. Formyl-CoA is rapidly hydrolysed to formic acid [14]. Pristanal is converted into pristanic acid in a reaction catalyzed by NAD ${ }^{+}$. F urther $\beta$-oxidation of pristanic acid requires activation to pristanoyl-CoA. 
suggest that the structure of the pathway is as outlined in fig. 3 with pristanal as obligatory intermediate. Current studies are directed towards identification of the enzymes involved, their subcellular localisation and their activity in tissues from patients suffering from peroxisomal disorders in which phytanic acid accumulates.

\section{REFERENCES}

1. J ansen, G. A., Mihalik, S. J ., Watkins, P. A., Moser, H. W., J akobs, C., Denis, S., and Wanders, R. J . A. (1996) Biochem. Biophys. Res. Commun. 229, 205- 210.

2. Mihalik, S. J ., Rainville, A. M., and Watkins, P. A. (1995) Eur. J . Biochem. 232, 545-551.

3. Croes, K., Casteels, M., De Hoffmann, E., Mannaerts, G. P., and van Veldhoven, P. P. (1996) Eur. J . Biochem. 240, 674- 683.

4. J ansen, G. A., Wanders, R. J. A., Watkins, P. A., and Mihalik, S. J . (1997) N. Eng. J . Med. [In press]

5. J ansen, G. A., Mihalik, S. J ., Watkins, P. A., Moser, H. W., J akobs, C., Heijmans, H. S. A., and Wanders, R. J . A. (1997) J . Inher. Metab. Dis. [In press]

6. Verhoeven, N. M., Schor, D. S. M., Roe, C. R., Wanders, R. J . A., and J akobs, C. (1997) Biochim. Biophys. Acta [In press]
7. Sweetman, L., and Williams, J . C. (1995) in The Metabolic and Molecular Bases of Inherited Disease (Scriver, C. R., Beaudet, A. L., Sly, W. S., and Valle, D., Eds.), pp. 1387-1422, McGrawHill, New York.

8. Mitchell, D. Y., and Petersen, D. R. (1989) Arch. Biochem. Biophys. 269, 11- 17.

9. Brink, H.J . ten, Schor, D. S. M., Kok, R. M., Poll-The, B. T., Wanders, R. J . A., and J akobs, C. (1992) J . Lipid Res. 33, 14491457.

10. Rasmussen, J . T., Borcher, T., and Knutsen, J . (1990) Biochem. J . 265, 849-855.

11. Smith, P. K., Krohn, R. I., Hermanson, G. T., Mallia, A. K., Gertner, F.H., Provenzano, M.D., Fujimoto, E. K., Goeke, N. M., Olson, B. J ., and Klenk, D. C. (1985) Anal. Biochem. 150, 7685.

12. Brink, H.J . ten, Stellaard, F., van den Heuvel, C. M. M., Kok, R. M., Schor, D. S. M., Wanders, R. J . A., and J akobs, C. (1992) J . Lipid Res. 33, 41-47.

13. Watkins, P. A., Howard, A. E., Gould, S. J ., Avigan, J ., and Mihalik, S. J . (1996) J . Lipid Res. 37, 2288- 2295.

14. Croes, K., Veldhoven, P. P. van, Mannaerts, G. P., and Casteels, M. (1997) FEBS Lett. 407, 197- 200.

15. Martin, R. O., and Stumpf, P. K. (1959) J . Biol. Chem. 234, 2548- 2554. 